\title{
Frühjahrsmeeting der young dentists: Implantologie in Abrechnung und Praxis
}

\author{
Das gut besuchte Frühjahrsmeeting der young dentists $\left(\mathrm{yd}^{2}\right)$ in Bonn stand ganz im Zeichen der Implan- \\ tologie. Zunächst ging es dabei um den im Studium immer noch sträflich vernachlässigten Bereich der \\ privaten Abrechnung, ehe am zweiten Tag praktisches Arbeiten bei einer Einführung in die \\ Implantologie - "Mein erstes Implantat" mit den „Tröltzsch-Brothers" - anstand.
}

\section{Dr. Christian Honert // Asbach Markus Brakel // Düsseldorf}

\begin{abstract}
Ans Eingemachte bei der GOZ
Schon vor dem Vortrag von Dr. Dr. Alexander Raff, Herausgeber des BEMA- und GOZ-Abrechnungskommentars Liebold/Raff/ Wissing, sollte den Teilnehmern bereits klar gewesen sein: Die genaue Kenntnis der relevanten Paragrafen der GOZ ist die absolute Basis für eine wirtschaftliche Praxisführung. „GOZ essentiell: die Struktur und das Wesen der privaten Gebührenordnung für Zahnärzte besser verstehen", so lautete das Vortragsthema.

Und darin ging es ans Eingemachte: Der Anwendungsbereich der privaten Gebührenordnung, die Inhalte und Vorschriften der abweichenden Vereinbarungen („freie Honorarvereinbarung“), das adäquate Bemessen der Gebührenhöhe („Faktorsteigerung“), die Analogberechnung nicht in der GOZ oder GOÄ enthaltener Leistungen, der korrekte Ansatz von Material- und Laborkosten sowie eine formal korrekte Rechnungsstellung waren die Themen, die eingehend besprochen und grundsätzlich durchdrungen werden sollten. Auch die Formvorschriften, die einzuhalten sind, um bei GKV-Patienten zusätzliche Privatleistungen anbieten zu können, waren Seminargegenstand.
\end{abstract}

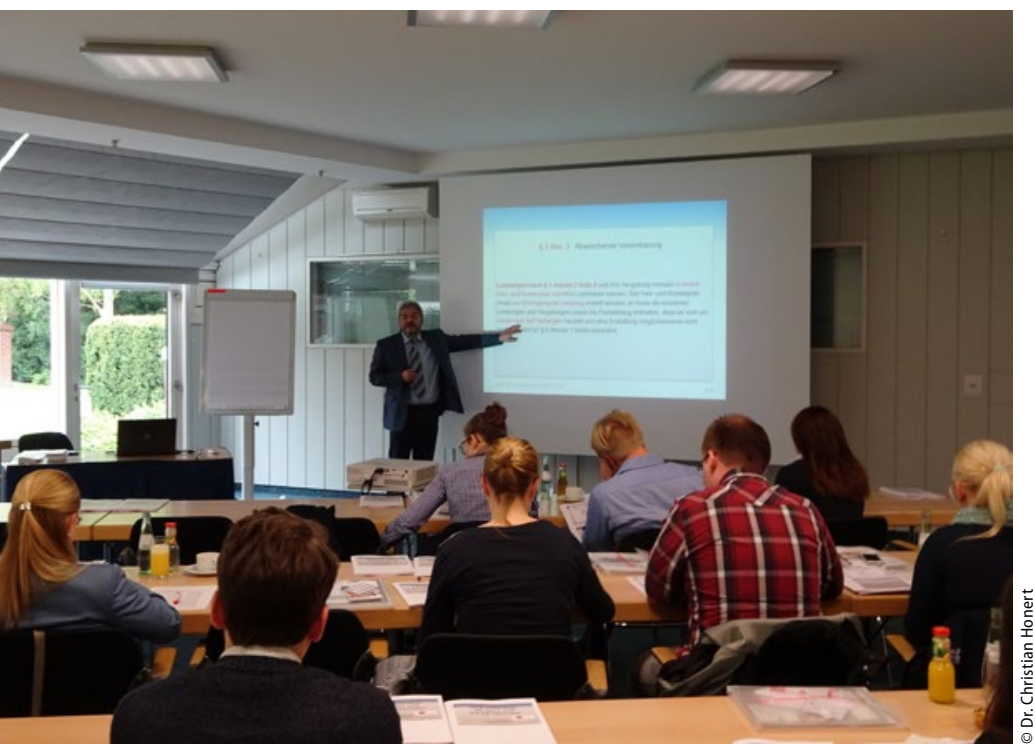

Ein eher trockenes Thema, das dennoch anregend vermittelt wurde: Referent Raff teilte sein Wissen zur privatzahnärztlichen Abrechnung.

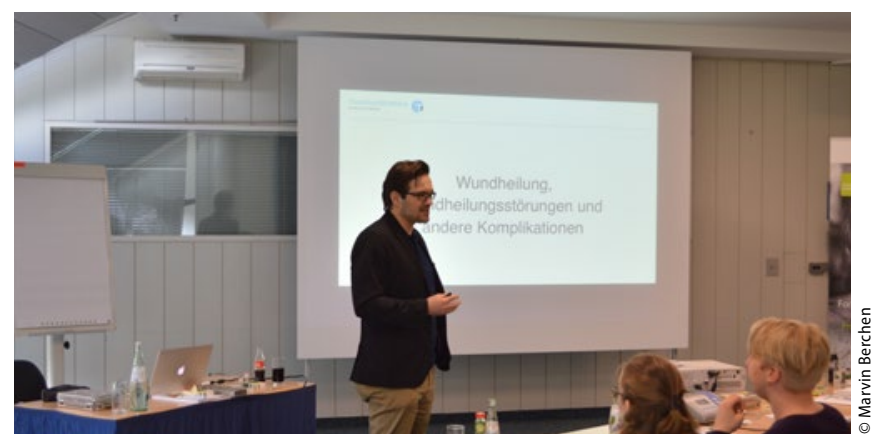

Dr. Dr. Markus Tröltsch beim Vortrag.

Da im wissenschaftlichen Teil des yd-Meetings implantologische Themen erörtert werden sollten, wurden die Grundlagen einer GOZ-konformen und betriebswirtschaftlich korrekten Liquidation anhand implantologischer und implantatprothetischer Gebührennummern und Fallbeispielen im zweiten Seminarteil konkret erläutert. So wurde die Wissenschaft wirkungsvoll mit der Praxisökonomie verknüpft.

Dazu gab es jede Menge praktische Beispiele, wie man eine korrekte Rechnung gestaltet und wie man richtig mit Behauptungen der Krankenkassen umgeht, wenn Leistungen durch die Krankenkasse willkürlich oder aber mittels falscher Behauptungen gekürzt werden. „Es ist zwar ein trockenens Thema, allerdings ist es dem Referenten Raff anhand der zahlreichen Beispiele sehr gut gelungen, die jungen Leute mitzunehmen“, befand einer der Teilnehmer.

\section{Mein erstes Implantat}

Mit den „Tröltzsch-Brothers“ Dr. Dr. Matthias und Dr. Dr. Markus Tröltzsch (beide MKG-Chirurgen, letzterer ist neuer Vorsitzender der Akademie und Wissenschaft - APW) folgte der Einstieg in die praktische Arbeit. „Mein erstes Implantat“ und der Weg dorthin standen im Folgenden auf der Agenda. Das reichte von anatomischem Grundlagenwissen bis hin $\mathrm{zu}$ den Grundlagen der Implantologie und Hands-on-Arbeiten. Es gab außerdem zahlreiche klinische Fälle zu sehen. An Orangen wurden Nahttechniken geübt, und Highlight war dann die Insertion eines Implantats im Kunststoffkiefer. „Auch die Vorträge der Tröltzsch-Brothers waren top“ - das war der Tenor zu einem gelungenen Exkurs in die Praxis.

Weitere Infos und einen Überblick über die angebotenen $\mathrm{yd}^{2}$-Seminare bietet die Homepage www.young-dentists.de. Das Herbstmeeting der $\mathrm{yd}^{2}$ findet am 29./30. September statt. 